\title{
Dynamic analysis of hydrate-bearing seabed sediments considering methane gas production induced by depressurization
}

\author{
T. Akaki ${ }^{\mathrm{i})}$, S. Kimoto ${ }^{\mathrm{ii})}$ and F. Oka ${ }^{\mathrm{iii})}$ \\ i) Ph.D. student, Department of Civil and Earth Resources Engineering, Kyoto University, 4 Kyoto Daigaku-Katsura, \\ Nishikyo-ku, Kyoto 615-8530, Japan. \\ ii) Associate Professor, Department of Civil and Earth Resources Engineering, Kyoto University, 4 Kyoto Daigaku-Katsura, \\ Nishikyo-ku, Kyoto 615-8530, Japan. \\ iii) Professor Emeritus, Kyoto University, 36-1 Yoshidahon-machi, Sakyo-ku, Kyoto 606-8501, Japan.
}

\begin{abstract}
Understanding the mechanical behavior of hydrate-bearing sediments during earthquakes is important for the safe and long term gas production from methane hydrates. Additionally, long term and widespread gas production may have effects on the mechanical properties of the seabed ground. In the present study we have analyzed the dynamic behavior of hydrate-bearing sediments considering gas production by the depressurization method. Firstly, we simulated gas production process using a chemo-thermo-mechanically coupled method for different levels of depressurizing pressure. Then, we conducted dynamic analyses by using the results of the production analyses as the initial conditions in order to consider the effects of gas production on dynamic behavior of the sediments. We have modeled the hydrate bearingsediments as a multiphase mixture composed of gas, water, soil, and hydrates. For the constitutive model for soils, we used a cyclic elasto-viscoplastic model for clayey soils with nonlinear kinematic hardening. A predicted earthquake motion at Kumanonada along the Nankai trough was input. From the numerical results, we have found that the production of natural gas may affect the ground motion during strong earthquakes.
\end{abstract}

Keywords: methane hydrate, earthquake, depressurization

\section{INTRODUCTION}

Nowadays methane hydrates are viewed as a new energy resource since they contain much developable methane gas. They are ice-like materials composed of methane and water which naturally exist in the permafrost sediments and the deep seabed ground. Recently, the world's first offshore production test from methane hydrates bearing sediments by the depressurization method has been conducted off the coasts of Atsumi and Shima peninsula in Japan. A volume of 120,000 $\mathrm{m}^{3}$ methane gas was recovered during 6 days.

There are currently several numerical models that can simulate the flow and deformation behavior during hydrate dissociation (Kimoto et al. 2007, 2010; Moridis et al. 2009; Sakamoto et al. 2009; Kurihara et al. 2011; White et al. 2008). From the numerical results, it has been reported that ground deformation will be induced by generation and dissipation of water and gas pressure around the hydrate dissociation area. There are, however, few studies on the dynamic behavior of hydrate-bearing sediments. Earthquake during methane gas production may damage production equipment and cause the ground deformation. Therefore, it is important to study the dynamic behavior of hydrate-bearing sediments for the safe and long term gas production. Additionally, long term and widespread gas production from hydrate bearing sediments may have effects on the mechanical properties of the ground. Hence the variation of mechanical properties of the site due to gas production should be also considered for earthquake during gas production. In the present study, we have analyzed the earthquake response of hydrate-bearing sediments during the methane gas production. Firstly, we have conducted simulations of the methane gas production by the depressurization with different levels of pressure using a chemo-thermo-mechanically coupled method (Kimoto et al. 2007, 2010). Then, we conducted dynamic analyses using the results of the simulations as the initial conditions in order to consider the effects of the production of methane gas by depressurization on the mechanical behavior of the sediments during earthquakes. 


\section{SIMULATION METHOD}

\subsection{Multiphase mixture theory}

Geomaterials generally fall into the category of multiphase materials which are basically composed of soil particles, water and air. The macroscopic mechanical behavior can be described within the framework of a continuum approach through the use of the theory of porous media. Shahbodagh (Shahbodagh et al. 2011, 2014) presented a computational framework for large deformation dynamic analysis for partially saturated elasto-viscoplastic soils. In the present study, we have extended the formulation to hydrate bearing sediments with temperature change by adding hydrate phase and considering the conservation of energy.

\subsection{Constitutive equations}

We used a cyclic elasto-viscoplastic model based on overstress type viscoplastic theory with the nonelinear kinematic hardening rules and the structural degradation for solid phase (Kimoto et al. 2013). In the model, we consider the hydrate saturation dependency on the stressstrain behavior by introducing a hardening parameter. The elastic behavior is given by a generalized Hooke type of law. The shear modulus, which is important for seismic response analysis, includes the stress and the viscoplastic strain dependency as follows:

$$
\frac{G}{G_{0}}=\frac{1}{1+\alpha^{\prime}\left(\gamma^{v p}\right)^{r}} \sqrt{\frac{\sigma_{m}^{\prime}}{\sigma_{m 0}^{\prime}}}
$$

where $G_{0}$ is the initial shear modulus, $\gamma^{v p}$ is the accumulated viscoplastic shear strain, $\sigma_{m}^{\prime}$ is the mean skeleton stress, $\sigma_{m 0}^{\prime}$ is the initial value of the mean skeleton stress, and $\alpha^{\prime}$ and $r$ are material parameters. We assumed the ideal gas for the gas phase and van Genuchten model is used for soil-water characteristic curve. Fig1. shows the schematic figure of the methane hydrate bearing soil and the governing equations. We assumed that hydrate bearing ground are usually composed of soil particles, hydrates, water and gas.
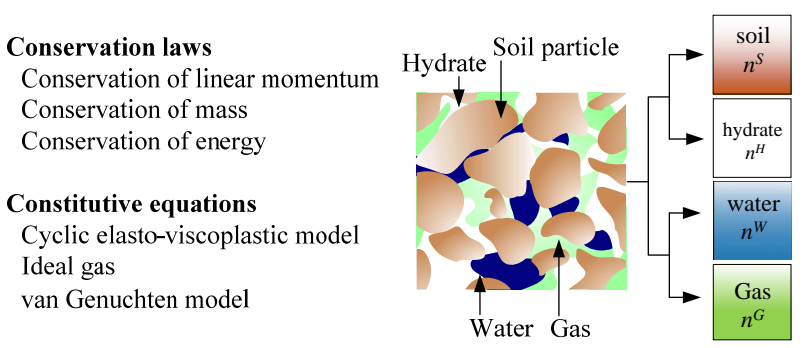

Fig.1 Methane hydrate baring soil and governing equations.

\subsection{Discretization of the governing equations}

Weak form of the mass conservations of mass, the conservation of momentum and the conservation of energy are discretized in space by the finite element method. Dealing with a large deformation, an updated Lagrangian method with the objective Jaumann rate of Cauchy stress is used. For the time discretization, Newmark's $\beta$ method is adopted. In addition, the damping of the system proportional to the initial stiffness matrix is considered in the analyses. The unknown variables are the acceleration of the solid phase, the pore water pressure, the pore gas pressure, and the temperature. In the present simulations, the dissociation during the dynamic analyses is not considered for simplicity.

\section{INITIAL AND BOUNDARY CONDITIONS}

Analyzing the dynamic response of hydrate-bearing sediments considering gas production effects on the mechanical properties, we used the results of simulation of gas production process as the initial conditions of the dynamic analyses. We used chemo-thermo-mechanically coupled method (Kimoto et al. 2007, 2010) for the simulation of gas production process. Ground deformation, water and gas flow, phase change from solid to fluid and the heat transfer occur during gas production from methane hydrates. This initial conditions include the change of physical quantities such as the stress, the pore water and the gas pressure, the temperature, the void ratio and the hydrate saturation.

Finite element mesh and the boundary conditions used in the simulations are displayed in Fig.2 The seabed ground at the water depth of $1000 \mathrm{~m}$ is modeled, and the horizontally layered ground under two-dimensional plane strain condition is assumed. We used an elastic model for elements up to $115 \mathrm{~m}$ from the bottom in depth, and a cyclic elasto-viscoplastic model for the other elements. There exists a hydrate-bearing sediment which has a thickness of $45 \mathrm{~m}$ at a depth of $290 \mathrm{~m}$ from the seabed. The equi-displacement is assumed for the nodes at the same depth. Viscous boundary is used on the bottom. Water pressure is set to be constant value of $9.8 \mathrm{MPa}$ and temperature is set to be constant value of $277 \mathrm{~K}$ at the top of the model ground. Constant water pressure and temperature are assumed on the top and bottom side.

The input ground motion is illustrated in Fig.3 A predicted earthquake motion at Kumanonada for the hypothetical great earthquake along the Nankai trough predicted (Sugito et al. 2000) is used in the simulation. 
The magnitude of the hypothetical earthquake is 9.0 and the epicenter is off the coast of Kii peninsula.

Simulation results of the cyclic undrained triaxial test by the elasto-viscoplastic constitutive model with the material parameters used in the dynamic analyses are presented in Fig.4. the effective confining pressure is $200 \mathrm{kPa}$, the cyclic stress ratio is 0.300 .The simulation ended when double amplitude of the axial strain reaches $16.0 \%$, that is, cyclic number is 17.5 in this case.

Fig. 5 shows the initial distribution of the pore water pressure, the mean skeleton stress and the temperature for the dynamic analyses which is obtained from the simulation of the production process for the different levels of depressurizing pressure of 0 (no production), 3 , 6 , and $9 \mathrm{MPa}$. The mean skeleton stress increases, the pore water pressure decreases due to the depressurization, and the temperature decreases in the hydrate-bearing layer due to the dissociation of hydrates.

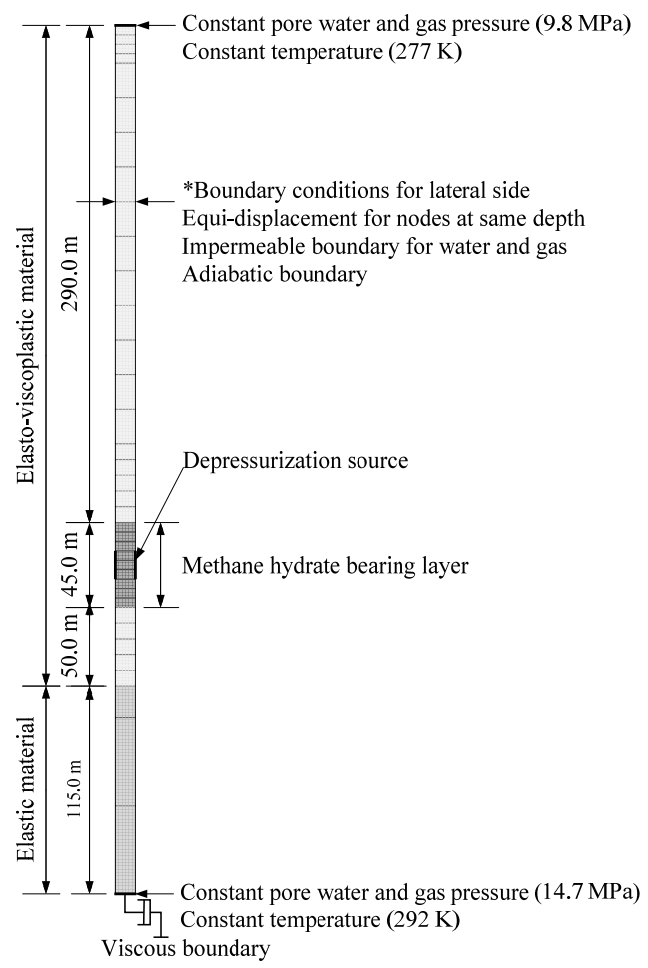

Fig.2 Finite element mesh for the dynamic analyses

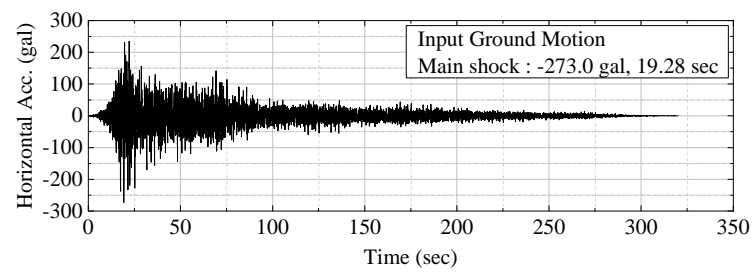

Fig.3 Input ground motion

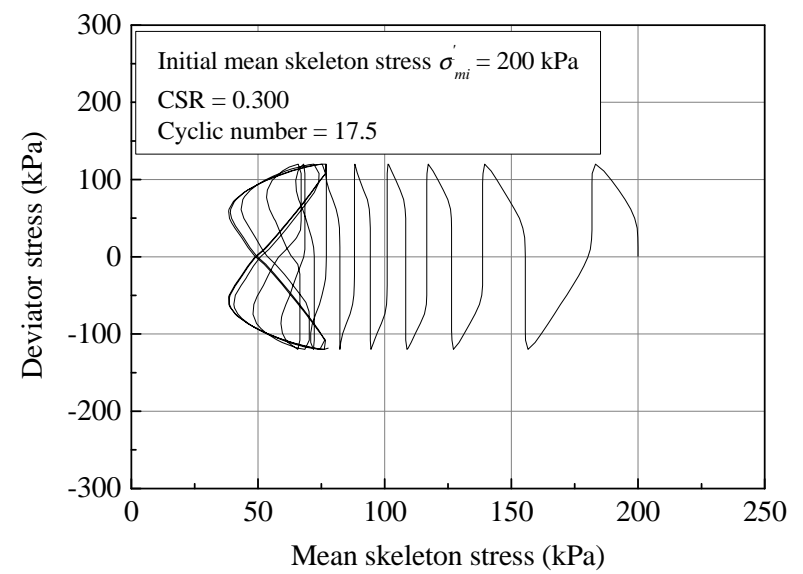

(a) Mean skeleton stress - deviator stress relation.

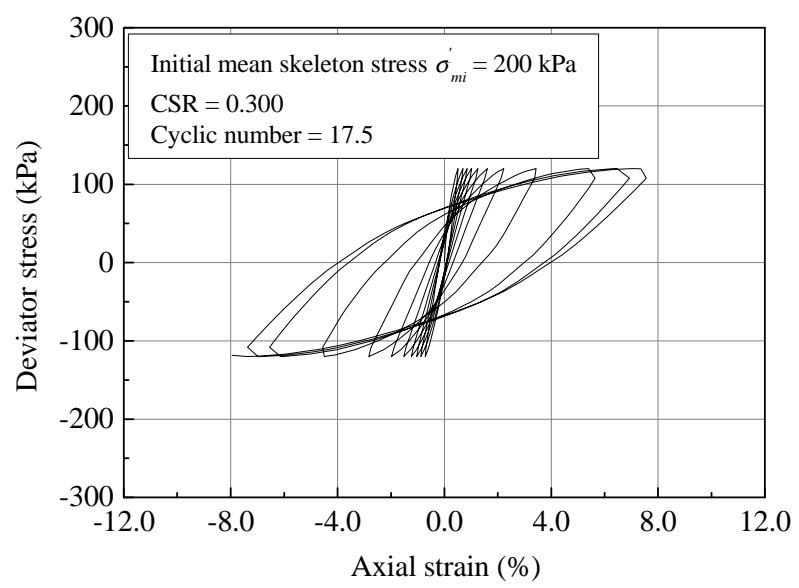

(b) Axial strain - deviator stress relation.

Fig.4 Simulation results of the cyclic undrained triaxial test for parameters used in the dynamic analyses.
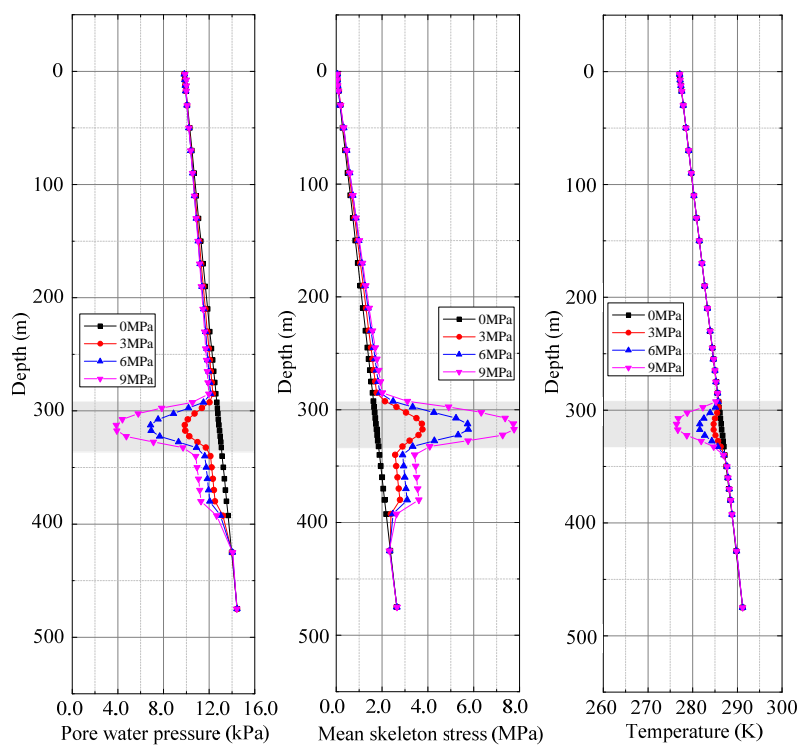

Fig.5 Distribution of the mean effective stress, pore water pressure and temperature before dynamic analyses 


\section{RESULTS OF DYNAMIC ANALYSIS}

\subsection{Horizontal acceleration and displacement}

Fig.6 presents the distribution of the maximum horizontal acceleration and the maximum horizontal displacement. The maximum horizontal acceleration shows different distributions from $200 \mathrm{~m}$ to $470 \mathrm{~m}$ in depth for the different depressurization pressure. The maximum horizontal acceleration in the hydrate bearing layer becomes larger for the case with gas production by depressurization than that for the case with no gas production. The maximum horizontal displacement for the cases with gas production show larger value than that of the case with no production. These differences may be related to the change of shear modulus associated with increase of skeleton stress due to the methane gas production.
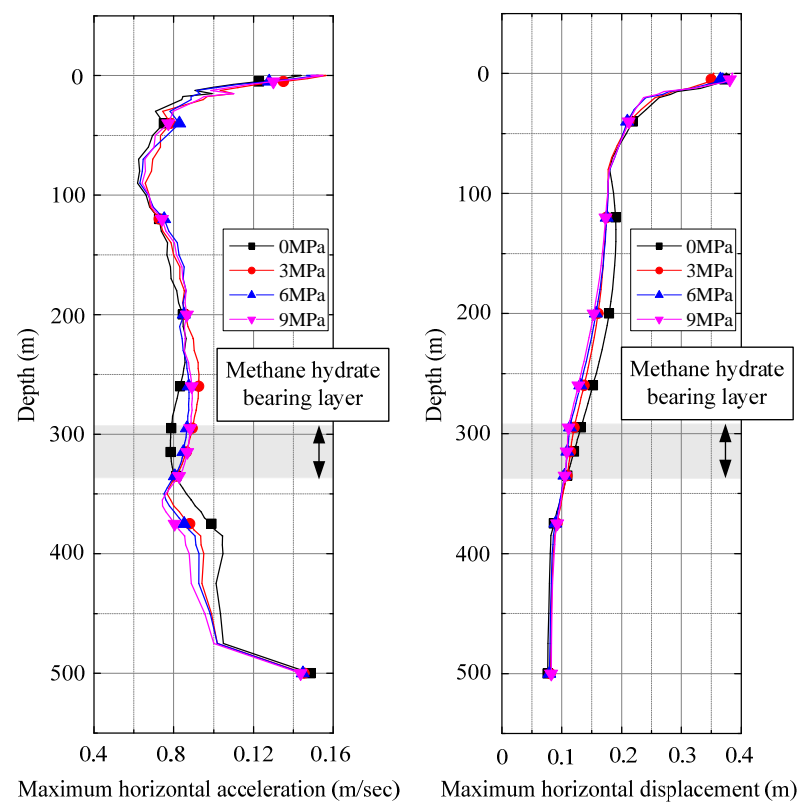

Fig.6 Distributions of maximum horizontal acceleration and the maximum horizontal displacement.

\subsection{Stress path and stress-strain relations}

The skeleton stress path and the stress-strain relations of element at a depth of $12.5 \mathrm{~m}$ are compared in Fig.7 for the depressurization pressures of $0 \mathrm{MPa}$ and $6 \mathrm{MPa}$. The skeleton stress is defined as the difference between the total stress and the average pore pressure, which equals to the Terzaghi's effective stress for the water saturated soil. Fig.7 (a) presents the skeleton stress path. The mean skeleton stress paths show different behavior at the early stage, that is, the mean skeleton stress tends to decrease earlier for the case with depressurization. Final value of the mean skeleton stress is same for the both cases. Fig.7 (b) shows the shear stress $\tau_{x y}$ - shear strain $\gamma_{x y}$ relations. The stress-strain relation shows similar behavior. The absolute value of shear strain for the case with depressurization is slightly large.

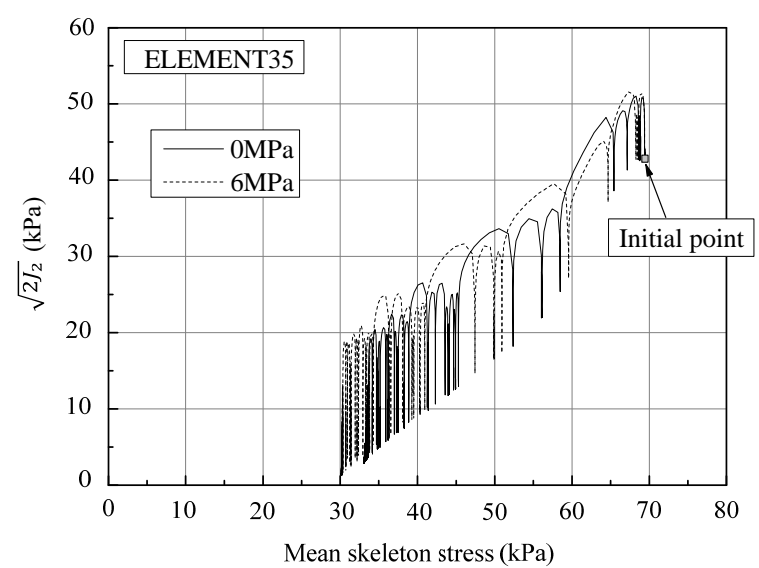

(a) Mean skeleton stress - second invariant of deviatric skeleton stress $\sqrt{2 J_{2}}$ relations.

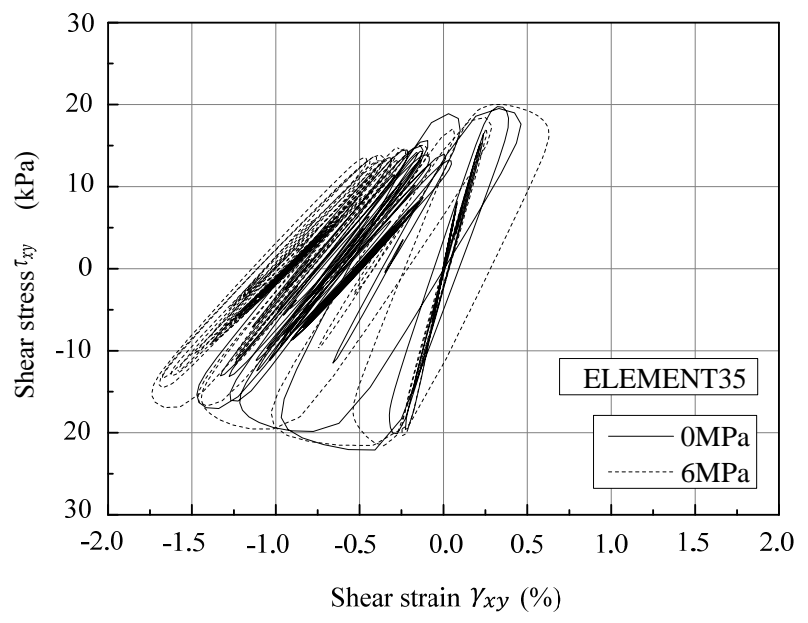

(b) Shear strain $\gamma_{x y}$ - shear stress $\tau_{x y}$ relations.

Fig.7 Stress paths and stress-strain relations of element 35.

\section{CONCLUSIONS}

We have developed a computational method for the large deformation dynamic analysis of partially saturated elasto-viscoplastic soils with methane hydrate. We have conducted dynamic analyses of hydrate-bearing sediments considering the effects of methane gas production by depressurization method on mechanical properties of seabed ground. From the numerical results, we have found that the maximum values of the horizontal acceleration showed different tendency for the different levels of depressurization pressure. The stress paths and the stress-strain relations of the element close to the 
seabed showed overall similar behavior and gave a small difference in the decrease of the mean skeleton stress at the early stage of earthquake. More studies are needed for the different ground profiles. In addition, it is necessary to prove the dynamic behavior with the simultaneous dissociation.

\section{REFERENCES}

1) Kimoto, S., Oka, F., Fushita, T., \& Fujiwaki, M. (2007): A chemo-thermo-mechanically coupled numerical simulation of the subsurface ground deformations due to methane hydrate dissociation, Computers and Geotechnics, 34(4), 216-228.

2) Kimoto, S., Oka, F., \& Fushita, T. (2010): A chemo-thermomechanically coupled analysis of ground deformation induced by gas hydrate dissociation, International Journal of Mechanical Sciences, 52(2), 365-376.

3) Moridis, G.J., Collett, T.S., Boswell, R., Kurihara, M., Reagan, M.T., Koh, C. (2009): Toward Production From Gas Hydrates: Current Status, Assessment of Resources, and Simulation-Based Evaluation of Technology and Potential, Proceedings of SPE Unconventional Reservoirs Conferrence, Lawrence Berkeley National Laboratory, Keystone.

4) Sakamoto, Y., Kakumoto, M., Miyazaki, K., Tenma, N., Komai, T., Yamaguchi, T. (2009): Numerical Study on Dissociation of Methane Hydrate and Gas Production Behavior in Laboratory-Scale Experiments for Depressurization: Part 3-Numerical Study on Estimation of Permeability in Methane Hydrate Reservoir, International Journal of Offshore and Polar Engineering, 19, 124-134.

5) Kurihara, M., Ouchi, H., Narita, H., Masuda, Y. (2011, July): Gas production from methane hydrate reservoirs, Proceedings of 7th International Conference on Gas Hydrates (ICGH). Edinburgh, UK.

6) White, M.D., McGrail, B.P. (2008): Numerical simulation of methane hydrate production from geologic formations via Carbon Dioxide injection, Proceedings of Offshore Technology Conference, Curran Associates, Reliant centre, Houston, Texas, USA, 1783-1794.

7) Shahbodagh, K.B. (2011): Large deformation analysis method for partially saturated elasto-viscoplastic soils, Ph.D. thesis, Kyoto University.

8) Shahbodagh Khan, B., Mirjalili, M., Kimoto, S., \& Oka, F. (2014): Dynamic analysis of strain localization in water saturated clay using a cyclic elasto - viscoplastic model, International Journal for Numerical and Analytical Methods in Geomechanics, 38(8), 771-793.

9) Kimoto, S., Shahbodagh, K.B., Mirjalili, M., Oka, F. (2013): A cyclic elasto-viscoplastic constitutive model for clay considering the nonlinear kinematic hardening rules and the structural degradation, International Journal of Geomechanics, 10.1061/(ASCE)GM.1943-5622.0000327.

10) Sugito, M., Furumoto, Y., \& Sugiyama, T. (2000, January): Strong motion prediction on rock surface by superposed evolutionary spectra, Proceedings of 12th world conference on earthquake engineering, 2111(4). 